they may give some satisfaction to those who have delved in the theory of those transformations, since they make the transformations appear, not as separate chapters of the analysis, but as special applications of formulas now well established in other parts of the theory. It is even possible to make a satisfactory and not over complicated sufficiency proof for a weak minimum, without the use of the Weierstrass notion of a field. For the Weierstrass formula can be proved directly with some ease for the second variation when once it has been seen to hold true for a conjugate system of solutions of Jacobi's equations. It is not strange that the second variation has not been attacked from this standpoint before in spite of the fact that in my recent review of the literature I have found several suggestions which might have instigated one to attempt it. The real reason is, I think, that the advances of Weierstrass and Hilbert were published after 1900 and about the time that Kneser found his envelope theorem. The tendency since then has been to discard the theory of the second variation in favor of the more geometrical theory, but the experiment, so far as I know, has not been completely successful.

The University of Chicago, December, 1919.

\title{
GROUPS GENERATED BY TWO OPERATORS OF ORDER THREE WHOSE PRODUCT IS OF ORDER FOUR.
}

\author{
BY PROFESSOR G. A. MILLER.
}

(Read before the American Mathematical Society December 30,1919.)

\section{§1. Introduction.}

IT is known that the only groups which are completely determined by the orders of two generators and the order of their product are the dihedral groups and the groups of movements of the five regular solids known to the ancients. In all other cases two generators which are not restricted except as regards their orders and the order of their product 
may give rise to any one of an infinite system of groups.* In particular, there is no upper limit to the number of distinct groups that can be generated by two operators of order three whose product is of order four. The group of smallest order which two such operators can generate is the well known group of order 24 which does not contain any subgroup of order 12, and it is known that this group is defined by the facts that it is generated by two operators of order 3 whose product is of order 4 provided that the square of this product is invariant under each of these two operators of order 3 .

In the present article we shall consider the groups generated by $s_{1}$ and $s_{2}$ when these operators satisfy the following conditions:

$$
s_{1}^{3}=s_{2}^{3}=\left(s_{1} s_{2}\right)^{4}=\left(s_{1}^{2} s_{2}\right)^{k}=1 \quad(k=3,4,5) .
$$

It is unnecessary to consider the case where $k=2$, since the equations $s_{1}^{3}=s_{2}{ }^{3}=\left(s_{1}{ }^{2} s_{2}\right)^{2}=1$ define the tetrahedral group and hence $s_{1} s_{2}$ could not be of order 4 . When $k=1$ the order of $s_{1} s_{2}$ could evidently not be 4 . Hence $3,4,5$ are the smallest positive integral values of $k$ when $s_{1} s_{2}$ is of order 4 .

\section{§ 2. The Order of $s_{1}^{2} s_{2}$ is Three.}

From the condition that $\left(s_{1}^{2} s_{2}\right)^{3}=1$ it follows that

$$
s_{1}^{2} s_{2} s_{1}^{2}=s_{2}^{2} s_{1} s_{2}^{2}, \quad s_{1} s_{2}^{2} s_{1}=s_{2} s_{1}^{2} s_{2} .
$$

Hence the two operators of order $4, s_{1} s_{2}$ and $s_{2} s_{1}$, are commutative and generate a group whose order cannot exceed 16 . This group must be invariant under the group $G$ generated by $s_{1}$ and $s_{2}$ since

$$
s_{1}^{2} s_{2} s_{1}^{2}=s_{1}^{2} s_{2}^{2} \cdot s_{2}^{2} s_{1}^{2} \text { and } s_{2}^{2} s_{1} s_{2}^{2}=s_{2}^{2} s_{1}^{2} \cdot s_{1}^{2} s_{2}^{2} \text {. }
$$

The order of $G$ can therefore not exceed 48 . That the order of $G$ cannot be less than 48 when no additional restrictions are imposed on $s_{1}$ and $s_{2}$ results directly from the fact that the abelian group of order 16 and of type $(2,2)$ admits an automorphism of order 3 in which no operator except identity corresponds to itself. Hence the theorem:

If two operators $s_{1}, s_{2}$ satisfy the conditions

$$
s_{1}^{3}=s_{2}^{3}=\left(s_{1}^{2} s_{2}\right)^{3}=\left(s_{1} s_{2}\right)^{4}=1
$$

but are not otherwise restricted, they generate the group of order

* G. A. Miller, Amer. Journal of Mathematics, vol. 24 (1902), p. 96. 
48 obtained by extending the abelian group of order 16 and of type $(2,2)$ by means of an operator of order 3 which transforms this group into itself but is not commutative with any of its operators besides identity.

This group of order 48 evidently contains 32 operators of order 3,12 operators of order 4 , and 3 operators of order 2 in addition to identity. Each operator of order 3 is commutative with its own powers only, while each of the other operators besides identity is commutative with exactly 16 operators of the group.

§ 3. The Order of $s_{1}{ }^{2} s_{2}$ is Four.

It is easy to see that when $s_{1}{ }^{2} s_{2}$ is of order 4 the order of $G$ is divisible by 168 since the two substitutions $s_{1}=a b c \cdot d e f$, $s_{2}=a e h \cdot c d g$ satisfy the given conditions, as results from the following equations:

$$
s_{1} s_{2}=a b d h \cdot c e f g, \quad s_{1}^{2} s_{2}=a d f h \cdot b e g c .
$$

These two substitutions generate a transitive substitution group of degree seven whose order is a multiple of 168 since $s_{1}^{2} s_{2} s_{1} s_{2}{ }^{2}=b f e d c g h$. The fact that the order of $s_{1}^{2} s_{2} s_{1} s_{2}{ }^{2}$ is actually 7 when $s_{1}, s_{2}$ represent two abstract operators which satisfy the conditions

$$
s_{1}{ }^{3}=s_{2}{ }^{3}=\left(s_{1} s_{2}\right)^{4}=\left(s_{1}{ }^{2} s_{2}\right)^{4}=1
$$

but are not otherwise restricted results from the following equations:

$$
\begin{aligned}
& \left(s_{1}^{2} s_{2} s_{1} s_{2}^{2}\right)^{7} \\
& =s_{1}^{2} s_{2} s_{1} s_{2}{ }^{2} s_{1}{ }^{2} s_{2} s_{1} s_{2}{ }^{2} s_{1}{ }^{2} s_{2} s_{1} s_{2}{ }^{2} s_{1}{ }^{2} s_{2} s_{1} s_{2}{ }^{2} s_{1}{ }^{2} s_{2} s_{1} s_{2}{ }^{2} s_{1}{ }^{2} s_{2} s_{1} s_{2}{ }^{2} s_{1}{ }^{2} s_{2} s_{1} s_{2}{ }^{2} \\
& =s_{1}^{2} s_{2} s_{1}^{2} s_{2} s_{1} s_{2} s_{1} s_{2}{ }^{2} s_{1} s_{2}{ }^{2} s_{1} s_{2}{ }^{2} s_{1}{ }^{2} s_{2}{ }^{2} s_{1}{ }^{2} s_{2} s_{1}{ }^{2} s_{2} \text {. } \\
& s_{1}^{2} s_{2} s_{1} s_{2} s_{1} s_{2}{ }^{2} s_{1} s_{2}{ }^{2} s_{1} s_{2}^{2} s_{1}^{2} s_{2}{ }^{2} s_{1}{ }^{2} s_{2} s_{1}^{2} s_{2} s_{1} s_{2}{ }^{2} \\
& =s_{2}{ }^{2} s_{1} s_{2}{ }^{2} s_{1}{ }^{2} s_{2}{ }^{2} s_{1} s_{2} s_{1}{ }^{2} s_{2}{ }^{2} s_{1} s_{2} s_{1} s_{2}{ }^{2} s_{1}{ }^{2} s_{2}{ }^{2} \\
& =s_{2}{ }^{2} s_{1}{ }^{2} s_{2} s_{1} s_{2} s_{1}^{2} s_{2} s_{1}^{2} s_{2} s_{1}{ }^{2} s_{2}{ }^{2} s_{1}{ }^{2} s_{2} s_{1}{ }^{2} s_{2}{ }^{2} \\
& =s_{2}^{2} s_{1}^{2} s_{2} s_{1}^{2} s_{2} s_{1}^{2} s_{2} s_{1}^{2} s_{2}^{2}=1 \text {. }
\end{aligned}
$$

To verify that $s_{1}, s_{2}$ actually generate the simple group of order 168 it may be noted that well-known defining relations of this group are as follows:*

* L. E. Dickson, Linear Groups, 1901, p. 303. 


$$
T^{2}=1, \quad S^{7}=1, \quad(S T)^{3}=1, \quad\left(S^{4} T\right)^{4}=1 .
$$

Let $T=s_{2} s_{1} s_{2} s_{1}$ and $S=\left(s_{1}^{2} s_{2} s_{1} s_{2}^{2}\right)^{2}$. Hence $S^{4}=s_{1}^{2} s_{2} s_{1} s_{2}{ }^{2}$.

$$
\begin{aligned}
\left(S^{4} T\right)^{4}= & \left(s_{1}{ }^{2} s_{2} s_{1}{ }^{2} s_{2} s_{1}\right)^{4}=s_{1}{ }^{2} s_{2} s_{1}{ }^{2} s_{2}{ }^{2} s_{1}{ }^{2} s_{2}{ }^{2} s_{1}{ }^{2} s_{2}{ }^{2} s_{1}{ }^{2} s_{2} s_{1}=1 ; \\
(S T)^{3}= & s_{1}{ }^{2} s_{2} s_{1} s_{2}{ }^{2} s_{1}{ }^{2} s_{2} s_{1}{ }^{2} s_{2} s_{1} \cdot s_{1}{ }^{2} s_{2} s_{1} s_{2}{ }^{2} s_{1}{ }^{2} s_{2} s_{1}{ }^{2} s_{2} s_{1} \\
& \cdot s_{1}{ }^{2} s_{2} s_{1} s_{2}{ }^{2} s_{1}{ }^{2} s_{2} s_{1}{ }^{2} s_{2} s_{1} \\
= & s_{1}{ }^{2} s_{2} s_{1} s_{2}{ }^{2} s_{1}{ }^{2} s_{2} s_{1}{ }^{2} s_{2}{ }^{2} s_{1} s_{2}{ }^{2} s_{1}{ }^{2} s_{2} s_{1}{ }^{2} s_{2}{ }^{2} s_{1} s_{2}{ }^{2} s_{1}{ }_{2} s_{2} s_{1}{ }^{2} s_{2} s_{1} \\
= & s_{1}{ }^{2} s_{2} s_{1} s_{2} s_{1} s_{2}{ }^{2} s_{1} s_{2} s_{1} s_{2} s_{1} s_{2}{ }^{2} s_{1} s_{2} s_{1} s_{2} s_{1} s_{2}{ }^{2} s_{1}{ }^{2}=1 .
\end{aligned}
$$

It has now been proved that $s_{1}{ }^{2} s_{2} s_{1} s_{2}{ }^{2}$ and $s_{2} s_{1} s_{2} s_{1}$ generate the simple group of order 168. This is also the group generated by $s_{1}$ and $s_{2}$ since

$$
\begin{aligned}
s_{1}{ }^{2} s_{2} s_{1} s_{2}{ }^{2} \cdot s_{2} s_{1} s_{2} s_{1} & =s_{1}{ }^{2} s_{2} s_{1}{ }^{2} s_{2} s_{1}=s_{2}{ }^{2} s_{1} s_{2}{ }^{2} s_{1}{ }^{2} \\
s_{2} s_{1} s_{2} s_{1} \cdot s_{1}{ }^{2} s_{2} s_{1} s_{2}{ }^{2} & =s_{2} s_{1} s_{2}{ }^{2} s_{1} s_{2}{ }^{2}=s_{2}{ }^{2} s_{1}{ }^{2} s_{2} s_{1}{ }^{2} \\
s_{1} s_{2} s_{1}{ }^{2} s_{2} \cdot s_{2}{ }^{2} s_{1}{ }^{2} s_{2} s_{1}{ }^{2} & =s_{1} s_{2} s_{1} s_{2} s_{1}{ }^{2} \\
s_{1} s_{2} s_{1} s_{2} s_{1}{ }^{2} \cdot s_{1}{ }^{2} s_{2} s_{1} s_{2}{ }^{2} & =s_{1} s_{2} s_{1} s_{2} s_{1} s_{2} s_{1} s_{2} \cdot s_{2}=s_{2} .
\end{aligned}
$$

Since the group generated by $s_{1}^{2} s_{2} s_{1} s_{2}^{2}$ and $s_{2} s_{1} s_{2} s_{1}$ contains $s_{2}$ it contains also $s_{1} s_{2}{ }^{2} s_{1}{ }^{2}$ and $s_{1}{ }^{2} s_{2} s_{1}{ }^{2}$. The product of these operators is $s_{1} s_{2}^{2} s_{1} s_{2} s_{1}{ }^{2}=\left(s_{2}{ }^{2} s_{1}{ }^{2} s_{2} s_{1}{ }^{2}\right)^{-1} \cdot s_{1}{ }^{2}$. This proves that the group generated by $s_{1}^{2} s_{2} s_{1} s_{2}^{2}$ and $s_{2} s_{1} s_{2} s_{1}$ is identical with the group generated by $s_{1}$ and $s_{2}$. Hence the following theorem has been established: The simple group of order 168 is completely defined by the following equations:

$$
s_{1}{ }^{3}=s_{2}{ }^{3}=\left(s_{1} s_{2}\right)^{4}=\left(s_{1}{ }^{2} s_{2}\right)^{4}=1 .
$$

As is well known, W. Dyck gave in 1882 the following defining equations of this simple group*

$$
s_{1}^{7}=1, \quad s_{2}{ }^{3}=1, \quad\left(s_{1} s_{2}\right)^{2}=1, \quad\left(s_{1}^{5} s_{2}\right)^{4}=1 .
$$

$\S 4$. The Order of $s_{1}^{2} s_{2}$ is Five.

If $s_{1}=a c b \cdot d f e$ and $s_{2}=c d f$ it results that $s_{1} s_{2}=a d c b \cdot e f$ and $s_{1}{ }^{2} s_{2}=$ abdec. Hence it follows that $s_{1}, s_{2}$ when subjected to the additional condition that $s_{1}^{2} s_{2}$ is of order 5 generate a group $G$ which has the alternating group of degree 6 for a quotient group. L. E. Dickson gave the following defining relations for this quotient group $\dagger$ :

* Math. Annalen, vol. 20, p. 41.

† This Bulletin, vol. 9 (1903), p. 303. 


$$
t_{1}^{4}=t_{2}{ }^{3}=\left(t_{1}^{3} t_{2} t_{1} t_{2}^{2}\right)^{2}=\left(t_{1}^{2} t_{2}\right)^{4}=1 .
$$

Let $t_{1}=s_{2}^{2} s_{1} s_{2}{ }^{2}$ and $t_{2}=s_{1}$. It is easy to see that $t_{1}$ and $t_{2}$ generate $G$. In fact, the group generated by $t_{1}$ and $t_{2}$ contains $\left(s_{2}^{2} s_{1} s_{2}^{2} s_{1}\right)^{2}=s_{1}^{2} s_{2}$, and hence it contains $s_{1}$ and $s_{2}$. The order of $t_{1}$ is four since it is the transform of $s_{1} s_{2}$ by $s_{2}$. The fact that the order of $t_{1}^{2} t_{2}$ is also four results from the following equations:

$$
\begin{aligned}
& s_{2}^{2} s_{1} s_{2} s_{1} s_{2}^{2} s_{1} \cdot s_{2}^{2} s_{1} s_{2} s_{1} s_{2}^{2} s_{1} \cdot s_{2}^{2} s_{1} s_{2} s_{1} s_{2}^{2} s_{1} \cdot s_{2}{ }^{2} s_{1} s_{2} s_{1} s_{2}^{2} s_{1} \\
& s_{2} s_{1} s_{2}{ }^{2} s_{1} s_{2}{ }^{2} s_{1} s_{2} s_{1} \cdot s_{1}{ }^{2} s_{2} s_{1}{ }^{2} s_{2} s_{1}{ }^{2} s_{2} \cdot s_{2} s_{1} s_{2}{ }^{2} s_{1} \\
& =s_{2}^{2} s_{1} s_{2} s_{1} \cdot s_{1}^{2} s_{2} s_{1}^{2} s_{2} s_{1}^{2} s_{2} . \\
& =s_{2}{ }^{2} s_{1} s_{2}{ }^{2} s_{1}{ }^{2} s_{2} s_{1}{ }^{2} s_{2}{ }^{2} s_{1} s_{2}{ }^{2} s_{1} s_{2}{ }^{2} s_{1} s_{2}{ }^{2} s_{1}{ }^{2} s_{2} s_{1}{ }^{2} . \\
& s_{2}^{2} s_{1} s_{2}^{2} s_{1}=s_{2}{ }^{2} s_{1} s_{2}{ }^{2} s_{1}{ }^{2} s_{2} s_{1}{ }^{2} \cdot s_{1}{ }^{2} s_{2} s_{1}{ }^{2} s_{2} \cdot s_{2}{ }^{2} s_{1}{ }^{2} s_{2} s_{1}{ }^{2} s_{2}{ }^{2} s_{1} s_{2}{ }^{2} s_{1} \\
& =s_{2}^{2} s_{1} s_{2}{ }^{2} s_{1}{ }^{2} s_{2} s_{1} s_{2} s_{1} s_{2} s_{1}^{2} s_{2}{ }^{2} s_{1} s_{2}{ }^{2} s_{1}=s_{2}{ }^{2} s_{1} s_{2}{ }^{2} s_{1}{ }^{2} \cdot s_{1}{ }^{2} s_{2}{ }^{2} s_{1}{ }^{2} \cdot s_{1}{ }^{2} s_{2}{ }^{2} s_{1} s_{2}{ }^{2} s_{1} \\
& =1 \text {. }
\end{aligned}
$$

It remains to find the order of $t_{1}^{3} t_{2} t_{1} t_{2}{ }^{2}=s_{2} s_{1}^{2} s_{2} s_{1} s_{2}{ }^{2} s_{1} s_{2}{ }^{2} s_{1}{ }^{2}$. We shall first prove that the square of this operator is invariant under each of the operators $s_{1}$ and $s_{2}$. The following equations exhibit the fact that this square is invariant under $s_{2}$ :

$$
\begin{aligned}
& s_{1}^{2} s_{2} s_{1} s_{2}{ }^{2} s_{1} s_{2}{ }^{2} s_{1}^{2} s_{2} s_{1}^{2} s_{2} s_{1} s_{2}{ }^{2} s_{1} s_{2}{ }^{2} s_{1}{ }^{2} s_{2} \\
& =s_{1}^{2} s_{2}{ }^{2} s_{1}{ }^{2} s_{2} s_{1}{ }^{2} s_{2} s_{1} s_{2} s_{1}^{2} s_{2} s_{1} s_{2}^{2} s_{1} s_{2}{ }^{2} s_{1}{ }^{2} s_{2} \\
& =s_{2} s_{1} s_{2} s_{1} s_{2}^{2} s_{1}^{2} s_{2} \beta_{1} s_{2} s_{1}^{2} s_{2} s_{1} s_{2}^{2} s_{1} s_{2}{ }^{2} s_{1}{ }^{2} s_{2} \\
& =s_{2} s_{1} s_{2} s_{1}^{2} s_{2} s_{1} s_{2} s_{1} s_{2}{ }^{2} s_{1} s_{2} s_{1}{ }^{2} s_{2} s_{1} s_{2}{ }^{2} s_{1} s_{2}{ }^{2} s_{1}{ }^{2} s_{2} \\
& =s_{2} s_{1}^{2} s_{2}{ }^{2} s_{1} s_{2}{ }^{2} s_{1} s_{2}{ }^{2} s_{1}{ }^{2} s_{2} s_{1} s_{2}{ }^{2} s_{1} s_{2} s_{1}{ }^{2} s_{2} s_{1} s_{2}{ }^{2} s_{1} s_{2}{ }^{2} s_{1}{ }^{2} s_{2} \\
& =s_{2} s_{1}{ }^{2} s_{2}{ }^{2} s_{1} s_{2}{ }^{2} s_{1}{ }^{2} s_{2} s_{1} s_{2} s_{1} s_{2}{ }^{2} s_{1} s_{2}{ }^{2} s_{1} s_{2} s_{1}{ }^{2} s_{2} s_{1} s_{2}{ }^{2} s_{1} s_{2}{ }^{2} s_{1}{ }^{2} s_{2} \\
& =s_{2} s_{1}{ }^{2} s_{2}{ }^{2} s_{1}{ }^{2} s_{2} s_{1} s_{2} s_{1} s_{2}{ }^{2} s_{1} s_{2}{ }^{2} s_{1}{ }^{2} s_{2} s_{1}{ }^{2} s_{2}{ }^{2} s_{1}{ }^{2} s_{2} s_{1} s_{2}{ }^{2} s_{1} s_{2}{ }^{2} s_{1}{ }^{2} s_{2} \\
& =s_{2} s_{1}{ }^{2} s_{2} s_{1} s_{2}{ }^{2} s_{1} s_{2}{ }^{2} s_{1} s_{2}{ }^{2} s_{1}{ }^{2} s_{2} s_{1} s_{2}{ }^{2} s_{1} s_{2}{ }^{2} s_{1}{ }^{2} s_{2} s_{1}{ }^{2} s_{2}{ }^{2} s_{1}{ }^{2} s_{2} s_{1} s_{2}{ }^{2} s_{1} s_{2}{ }^{2} s_{1}{ }^{2} s_{2} \\
& =s_{2} s_{1}{ }^{2} s_{2} s_{1} s_{2}{ }^{2} s_{1} s_{2}{ }^{2} s_{1}{ }^{2} s_{2} s_{1} s_{2} s_{1} s_{2}{ }^{2} s_{1} s_{2}{ }^{2} s_{1} s_{2}{ }^{2} s_{1}{ }^{2} s_{2} s_{1}{ }^{2} s_{2}{ }^{2} s_{1}{ }^{2} s_{2} s_{1} s_{2}{ }^{2} s_{1} s_{2}{ }^{2} s_{1}{ }^{2} s_{2} \\
& =s_{2} s_{1}{ }^{2} s_{2} s_{1} s_{2}{ }^{2} s_{1} s_{2}{ }^{2} s_{1}{ }^{2} s_{2} s_{1} s_{2}{ }^{2} s_{1}{ }^{2} s_{2} s_{1} s_{2} s_{1}{ }^{2} s_{2}{ }^{2} s_{1}{ }^{2} s_{2}{ }^{2} s_{1}{ }^{2} s_{2} s_{1}{ }^{2} s_{2} s_{1} s_{2} \\
& =s_{2} s_{1}{ }^{2} s_{2} s_{1} s_{2}{ }^{2} s_{1} s_{2}{ }^{2} s_{1}{ }^{2} s_{2} s_{1}{ }^{2} s_{2} s_{1} s_{2} s_{1} s_{2}{ }^{2} s_{1} s_{2}{ }^{2} s_{1} s_{2}{ }^{2} s_{1}{ }^{2} s_{2} s_{1} s_{2} \\
& =s_{2} s_{1}{ }^{2} s_{2} s_{1} s_{2}{ }^{2} s_{1} s_{2}{ }^{2} s_{1}{ }^{2} s_{2} s_{1}{ }^{2} s_{2} s_{1} s_{2}{ }^{2} s_{1}{ }^{2} s_{2} s_{1} s_{2} s_{1} s_{2} \\
& =s_{2} s_{1}^{2} s_{2} s_{1} s_{2}{ }^{2} s_{1} s_{2}{ }^{2} s_{1}{ }^{2} s_{2} s_{1}{ }^{2} s_{2} s_{1} s_{2}{ }^{2} s_{1} s_{2}{ }^{2} s_{1}{ }^{2} \text {. }
\end{aligned}
$$

From the following equations it results that this square is also invariant under $s_{1}$. 
The transform of this square by $s_{1}$ is

$$
\begin{aligned}
& s_{1}^{2} s_{2} s_{1}^{2} s_{2} s_{1} s_{2}^{2} s_{1} s_{2}^{2} s_{1}^{2} s_{2} s_{1}^{2} s_{2} s_{1} s_{2}^{2} s_{1} s_{2}^{2} \\
& =s_{1}^{2} s_{2} s_{1} s_{2}^{2} s_{1}^{2} s_{2}^{2} s_{1}^{2} s_{2} s_{1} s_{2}^{2} s_{1}^{2} s_{2} s_{1}^{2} s_{2} s_{1} s_{2}^{2} s_{1} s_{2}^{2} \\
& =s_{1}^{2} s_{2} s_{1} s_{2}^{2} s_{1}^{2} s_{2} s_{1} s_{2}^{2} s_{1} s_{2}^{2} s_{1} s_{2}^{2} s_{1}^{2} s_{2}^{2} s_{1}^{2} s_{2} s_{1}^{2} s_{2} s_{1} s_{2}{ }^{2} s_{1} s_{2}{ }^{2} \\
& =s_{1}^{2} s_{2} s_{1} s_{2}^{2} s_{1} s_{2}^{2} s_{1}^{2} s_{2}^{2} s_{1}^{2} s_{2} s_{1} s_{2}^{2} s_{1}^{2} s_{2} s_{1} s_{2}^{2} s_{1}^{2} s_{2} s_{1} s_{2}^{2} s_{1} s_{2}^{2} \\
& =s_{1}^{2} s_{2} s_{1} s_{2}^{2} s_{1} s_{2}^{2} s_{1}^{2} s_{2} s_{1} s_{2}{ }^{2} s_{1} s_{2}^{2} s_{1} s_{2}^{2} s_{1}^{2} s_{2}^{2} s_{1}^{2} s_{2} s_{1} s_{2}^{2} s_{1}^{2} s_{2} s_{1} s_{2}{ }^{2} s_{1} s_{2}^{2} \\
& =s_{1}^{2} s_{2} s_{1} s_{2}^{2} s_{1} s_{2}^{2} s_{1}^{2} s_{2} s_{1} s_{2}^{2} s_{1} s_{2}^{2} s_{1}^{2} s_{2} s_{1} s_{2}^{2} s_{1} s_{2}^{2} s_{1}^{2} s_{2} s_{1} s_{2}^{2} s_{1} s_{2}^{2} \\
& =s_{1}^{2} s_{2} s_{1} s_{2}^{2} s_{1} s_{2}^{2} s_{1}^{2} s_{2} s_{1} s_{2}^{2} s_{1}^{2} s_{2} s_{1} s_{2} s_{1} s_{2}^{2} s_{1} s_{2}{ }^{2} s_{1} s_{2}^{2} s_{1}^{2} s_{2} s_{1} s_{2}^{2} s_{1} s_{2}^{2} \\
& =s_{1}^{2} s_{2} s_{1} s_{2}^{2} s_{1} s_{2}^{2} s_{1}^{2} s_{2} s_{1}^{2} s_{2} s_{1} s_{2} s_{1} s_{2}^{2} s_{1} s_{2}^{2} s_{1}^{2} s_{2} s_{1} s_{2} s_{1} s_{2}{ }^{2} s_{1} s_{2}{ }^{2} \\
& =s_{1}^{2} s_{2} s_{1} s_{2}^{2} s_{1} s_{2}^{2} s_{1}^{2} s_{2} s_{1}^{2} s_{2} s_{1} s_{2}^{2} s_{1}^{2} s_{2} s_{1}^{2} s_{2} s_{1} s_{2} s_{1} s_{2} s_{1} s_{2}^{2} s_{1} s_{2}^{2} \\
& =s_{1}^{2} s_{2} s_{1} s_{2}^{2} s_{1} s_{2}^{2} s_{1}^{2} s_{2} s_{1}^{2} s_{2} s_{1} s_{2}^{2} s_{1}^{2} s_{2} s_{1} s_{2} s_{1} s_{2}^{2} \\
& =s_{1}^{2} s_{2} s_{1} s_{2}^{2} s_{1} s_{2}^{2} s_{1}^{2} s_{2} s_{1}^{2} s_{2} s_{1} s_{2}^{2} s_{1} s_{2}^{2} s_{1}^{2} s_{2} \text {. }
\end{aligned}
$$

Having proved that this square is invariant under $s_{1}$ and $s_{2}$ we proceed to prove that the cube of this square is identity: $s_{2} s_{1}^{2} s_{2} s_{1} s_{2}^{2} s_{1} s_{2}^{2} s_{1}^{2} s_{2} s_{1}^{2} s_{2} s_{1} s_{2}^{2} s_{1} s_{2}^{2} s_{1}^{2}$

$\cdot s_{2} s_{1}^{2} s_{2} s_{1} s_{2}^{2} s_{1} s_{2}^{2} s_{1}^{2} s_{2} s_{1}^{2} s_{2} s_{1} s_{2}^{2} s_{1} s_{2}^{2} s_{1}^{2}$

$=s_{2} s_{1}^{2} s_{2} s_{1} s_{2}^{2} s_{1} s_{2}^{2} s_{1}^{2} s_{2} s_{1}^{2} s_{2} s_{1} s_{2}^{2} s_{1} s_{2}^{2} s_{1}^{2}$

$\cdot s_{1} s_{2}^{2} s_{1}^{2} s_{2} s_{1}^{2} s_{2} s_{1} s_{2}^{2} s_{1} s_{2}^{2} s_{1}^{2} s_{2} s_{1}^{2} s_{2} s_{1} s_{2}^{2}$

$=s_{2}^{2} s_{1} s_{2}^{2} s_{1} s_{2}^{2} s_{1}^{2} s_{2} s_{1}^{2} s_{2} s_{1} s_{2}^{2} s_{1} s_{2} s_{1}^{2} s_{2} s_{1}^{2} s_{2} s_{1} s_{2}^{2} s_{1} s_{2}^{2} s_{1}^{2} s_{2} s_{1}^{2}$

$=s_{1}^{2} s_{2} s_{1}^{2} s_{2} s_{1} s_{2} s_{1}^{2} s_{2} s_{1} s_{2}^{2} s_{1}^{2} s_{2}^{2} s_{1} s_{2}^{2} s_{1}^{2} s_{2}^{2} s_{1} s_{2}^{2} s_{1}^{2} s_{2} s_{1}^{2}$

$=s_{1}^{2} s_{2} s_{1} s_{2} s_{1}^{2} s_{2} s_{1} s_{2} s_{1}^{2} s_{2} s_{1}^{2} s_{2} s_{1} s_{2} s_{1}^{2} s_{2}^{2} s_{1}^{2} s_{2}^{2} s_{1} s_{2}^{2}$

$=s_{1}^{2} s_{2} s_{1} s_{2} s_{1} s_{2}^{2} s_{1}^{2} s_{2}^{2} s_{1} s_{2} s_{1}^{2} s_{2} s_{1} s_{2}^{2} s_{1} s_{2} s_{1}^{2} s_{2}^{2}$ $s_{1} s_{2}^{2} s_{1}^{2} s_{2} s_{1}^{2} s_{2}^{2} s_{1} s_{2} s_{1}^{2} s_{2} s_{1} s_{2}^{2} s_{1} s_{2} s_{1}^{2} s_{2}^{2}$

$\cdot s_{2} s_{1}^{2} s_{2} s_{1} s_{2}{ }^{2} s_{1} s_{2}{ }^{2} s_{1}{ }^{2} s_{2} s_{1}{ }^{2} s_{2} s_{1} s_{2}{ }^{2} s_{1} s_{2}{ }^{2} s_{1}{ }^{2}$

$=s_{2} s_{1}{ }^{2} s_{2} s_{1}^{2} s_{2}^{2} s_{1} s_{2} s_{1}^{2} s_{2} s_{1} s_{2}^{2} s_{1} s_{2} s_{1} s_{2} s_{1} s_{2}^{2} s_{1} s_{2}^{2} s_{1}^{2} s_{2} s_{1}^{2} s_{2} s_{1} s_{2}{ }^{2} s_{1}$

$=s_{1} s_{2}^{2} s_{1} s_{2}^{2} s_{1} s_{2} s_{1} s_{2} s_{1}^{2} s_{2} s_{1} s_{2} s_{1}^{2} s_{2} s_{1} s_{2}^{2} s_{1}^{2} s_{2} s_{1}^{2} s_{2} s_{1} s_{2}^{2} s_{1}$

$=s_{1}^{2} s_{2}^{2} s_{1} s_{2} s_{1}^{2} s_{2}^{2} s_{1} s_{2} s_{1} s_{2} s_{1}^{2} s_{2} s_{1} s_{2}^{2} s_{1}^{2} s_{2} s_{1}^{2} s_{2} s_{1} s_{2}^{2}$

$=s_{1}^{2} s_{2}^{2} s_{1} s_{2} s_{1}^{2} s_{2} s_{1}^{2} s_{2}^{2} s_{1} s_{2} s_{1} s_{2}^{2} s_{1}^{2} s_{2} s_{1}^{2} s_{2} s_{1} s_{2}^{2}$

$=s_{1}^{2} s_{2}^{2} s_{1} s_{2} s_{1}^{2} s_{2} s_{1}^{2} s_{2} s_{1}^{2} s_{2}^{2} s_{1}^{2} s_{2} s_{1}^{2} s_{2} s_{1}^{2} s_{2} s_{1} s_{2}^{2}$

$=s_{1}^{2} s_{2}^{2} s_{1}^{2} s_{2}^{2} s_{1}^{2} s_{2}^{2} s_{1}^{2} s_{2}^{2}=1$. 
Since $\left(t_{1}^{3} t_{2} t_{1} t_{2}^{2}\right)^{2}$ is invariant under $G$ and the order of this invariant operator is a divisor of 3 , it results that the order $g$ of $G$ must be a divisor of 1,080 . If $g=1,080, G$ can be represented as a transitive substitution group of degree 18 since it contains the icosahedral group, as results from the fact that

$$
s_{1}^{2} s_{2} \text { and } s_{1} s_{2} s_{1} s_{2}
$$

are two operators of orders 5 and 2 respectively whose product is of order 3 , since

$$
s_{1}^{2} s_{2} s_{1} s_{2} s_{1} s_{2} s_{1}{ }^{2} s_{2} s_{1} s_{2} s_{1} s_{2} s_{1}{ }^{2} s_{2} s_{1} s_{2} s_{1} s_{2}=s_{1} s_{2}{ }^{2} s_{1}{ }^{6} s_{2}{ }^{2} s_{1}{ }^{6} s_{2}{ }^{2} s_{1}{ }^{2}=1 .
$$

The subgroup of order 60 composed of all the substitutions which omit one letter of this transitive group is of degree 15 since $G$ contains an invariant operator of order 3 , and the six systems of intransitivity of the two invariant substitutions of order 3 of this transitive group are permuted according to the alternating group of degree 6 . If this subgroup is transitive, it is completely determined since the icosahedral group can be represented in essentially only one way as a transitive substitution group of degree 15 . That is, all such possible representations are conjugate substitution groups. Moreover, this transitive subgroup determines five cycles of the two invariant substitutions of order 3 contained in $G$.

If we assume that this subgroup is generated by the following substitutions:

$$
a b c \cdot d k f \cdot e n h \cdot g o i \cdot j l m, \quad a l \cdot b n \cdot c f \cdot d o \cdot g m \cdot h i,
$$

then the invariant substitutions of the substitution group of degree 18 which is simply isomorphic with $G$ may be assumed to be

$$
\begin{aligned}
& a b c \cdot d m h \cdot e k j \cdot f l n \cdot g i o \cdot p q r \\
& a c b \cdot d h m \cdot \text { ekj } \cdot f n l \cdot g o i \cdot p r q \cdot
\end{aligned}
$$

An additional generator of this substitution group is of order 4 and has for its square any one of the substitutions of order 2 contained in it, since all of these substitutions of order 2 are conjugate under this substitution group. As this substitution of order 4 is also commutative with the given invariant substitutions of order 3 and must involve three cycles of order 4 and three transpositions, it must be one of three substitutions. Assuming that its square is 


$$
d j \cdot e m \cdot f i \cdot g n \cdot h k \cdot l o,
$$

these three substitutions are as follows:

$$
\begin{aligned}
& a p \cdot b q \cdot c r \cdot d f j i \cdot m l e o \cdot h n k g \\
& a p \cdot b q \cdot c r \cdot d l j o \cdot m n e g \cdot h f k i \\
& a p \cdot b q \cdot c r \cdot d n j g \cdot m f e i \cdot h l k o .
\end{aligned}
$$

It is not difficult to verify that the first two of these substitutions together with the above group of order 60 and degree 15 give rise to substitutions which could not appear in the group under consideration. On the other hand, the last one of these three substitutions together with the group of order 60 actually generates a group of order 1,080 and of degree 18 . This group is generated by the following substitutions:

$$
\begin{aligned}
& s_{1}=a l k \cdot b n j \cdot c f e \cdot d m h \cdot g o i, \\
& s_{2}=a p k \cdot b q j \cdot c r e \cdot d i f \cdot g n h \cdot l m o .
\end{aligned}
$$

The fact that these substitutions satisfy the conditions imposed on $s_{1}$ and $s_{2}$ results from the following equations:

$$
\begin{aligned}
s_{1} s_{2} & =\text { amgl } \cdot \text { bhin } \cdot \text { cdof } \cdot e r \cdot j q \cdot k p, \\
s_{1}{ }^{2} s_{2} & =d g f r e \cdot h o n q j \cdot i l p k m .
\end{aligned}
$$

The given substitutions satisfy also the conditions imposed on $t_{1}$ and $t_{2}$, since

$$
\begin{aligned}
t_{1} & =a k \cdot b j \cdot c e \cdot d r i l \cdot f h q g \cdot m p o n, \\
t_{1}{ }^{2} t_{2} & =a l r k \cdot b n p j \cdot c f q l \cdot d g \cdot h o \cdot i m, \\
t_{1}{ }^{3} t_{2} t_{1} t_{2}{ }^{2} & =a l c f b n \cdot d k h e m j \cdot g i o \cdot p q r .
\end{aligned}
$$

The group generated by $s_{1}$ and $s_{2}$ contains

$$
\left(s_{1} s_{2} s_{1}\right)^{2}\left(s_{1} s_{2}\right)^{2} s_{1}{ }^{2} s_{2}=d h m \cdot e n o \cdot f g k \cdot i j l \cdot p q r .
$$

As the product of this substitution of order 3 and $s_{1}{ }^{2} s_{2}$ is of order 2, these two substitutions generate a transitive group of order 60 and of degree 15 . It therefore results that $s_{1}$ and $s_{2}$ generate a transitive group of degree 18 whose order cannot be less than 1,080, and from the given abstract properties of $s_{1}$ and $s_{2}$ it follows that this order cannot exceed 1,080 . It has therefore been proved that the $G$ is actually of order 1,080 . 
In the given group of order 60 each substitution of order 3 is transformed into itself by its own powers only. Hence in the group of order 1,080 such a substitution is transformed into itself by only 9 substitutions. The Sylow subgroups of order 27 contained in this group must therefore be nonabelian. Hence it results that $G$ does not contain a subgroup which is simply isomorphic with the alternating group of degree 6 .

It may be noted that to every operator in the central quotient group there corresponds at least one operator of $G$ whose order is equal to the order of the operator of this quotient group, and yet $G$ does not involve any subgroup which is simply isomorphic with the central quotient group. In this respect the present group differs from the group of order 120 which has for its central quotient group the icosohedral group but does not contain the latter as a subgroup. In this group of order 120, operators of order 4 correspond to operators of order 2 in the central quotient group. The Sylow subgroups of order 8 contained in $G$ are separately simply isomorphic with the octic group, just as in the simple group of order 360 , while the Sylow subgroups of order 27 are separately simply isomorphic with the non-abelian group of this order which involves no operator of order 9 . In particular, the following theorem has been established: There are two and only two groups, besides identity, which are generated by $s_{1}$ and $s_{2}$ when these two operators satisfy the conditions

$$
s_{1}^{3}=s_{2}{ }^{3}=\left(s_{1} s_{2}\right)^{4}=\left(s_{1}{ }^{2} s_{2}\right)^{5}=1 .
$$

One of these is the simple group of order 360 and the other is a group of order 1,080 whose central quotient group is this simple group but which does not involve this simple group as a subgroup.

UNIVERSITY OF ILLINOIS. 